\title{
Descriptive Report of Shoulder Range of Motion and Rotational Strength 6 and 12 Weeks Following Rotator Cuff Repair Using a Mini-Open Deltoid Splitting Technique
}

\author{
Todd S. Ellenbecker, PT, MS, SCS, OCS, CSCS ${ }^{1}$ \\ Eric Elmore, $\mathrm{MPT}^{2}$ \\ David S. Bailie, $M D^{3}$
}

Study Design: Retrospective chart review.

Objectives: To measure short-term postsurgery glenohumeral internal rotation and external rotation strength, shoulder range of motion (ROM), and subjective self-report ratings following mini-open rotator cuff repair of full-thickness rotator cuff tears.

Background: Physical therapists provide rehabilitation for patients following mini-open rotator cuff repair. Long-term outcome studies have reported a high percentage of good and excellent results following surgery; however, little has been published regarding the immediate short-term results of this procedure, during which the patient is under the direct care of the physical therapist.

Materials and Methods: Charts from 11 female and 26 male patients, with a mean \pm SD age of $57.3 \pm 9.9$ years, were reviewed following rotator cuff repair, using an arthroscopically assisted mini-open deltoid-splitting approach. All patients underwent postsurgery rehabilitation by the same therapist using a standard protocol. Retrospective chart review was used to obtain descriptive profiles of shoulder joint ROM at 6 and 12 weeks postsurgery and isokinetically assessed shoulder strength at 12 weeks postsurgery.

Results: For the postsurgical shoulder, ROM deficits ranging between $5^{\circ}$ to $7^{\circ}$ were measured for shoulder abduction and external rotation and internal rotation at $90^{\circ}$ of abduction. The postsurgical extremity had greater flexion ROM $\left(9^{\circ}\right)$ compared to the contralateral side. Isokinetic external rotation strength deficits of $5 \%$ to $7 \%$ were present at 12 weeks postsurgery, with $2 \%$ to $11 \%$ greater internal rotation shoulder strength on the operative extremity, when compared to the other side. Patients completed the self-report section of the modified American Shoulder Elbow Surgeons (ASES) Rating Scale at 12 weeks postsurgery and scored a mean of 38.7/45.0 points. Conclusion: The application of early ROM and progressive strengthening following mini-open rotator cuff repair allows for the successful return of ROM and strength 12 weeks postsurgery. The results of this study provide objective data for both shoulder ROM and strength at time points during which patients are traditionally receiving physical therapy following surgery. J Orthop Sports Phys Ther 2006;36(5):326-335. doi:10.2519/jospt.2006.2191

Key Words: glenohumeral joint, rotator cuff tear, surgery

1 Clinic Director, Physiotherapy Associates Scottsdale Sports Clinic, Scottsdale, AZ.

2 Physiotherapist, Physiotherapy Associates Paradise Valley Sports Clinic, Phoenix, AZ.

${ }^{3}$ Vice President, The Orthopedic Clinic Association, Phoenix, AZ.

Address correspondence to Todd S. Ellenbecker, Physiotherapy Associates Scottsdale Sports Clinic, 9917 N 95th Street, Scottsdale, AZ 85258. E-mail: ellenbeckerpt@cox.net

No research grant monies were used for this research investigation. Research was conducted at Physiotherapy Associates Scottsdale Sports Clinic and approved by the Institutional Review Board of Physiotherapy Associates, Memphis, TN. We affirm that we have no financial affiliation or involvement with any commercial organization that has direct financial interest in any matter included in this manuscript.

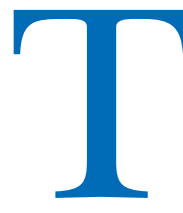

he provision of rehabilitation following rotator cuff repair is commonly performed by both orthopaedic and sports physical therapists. The use of the mini-open deltoid splitting surgical approach has been evaluated in the orthopaedic surgical literature with high percentages of good and excellent results reported, using subjective rating scales and in some cases objective measures of both range of motion and strength in short- and longterm follow-up studies. 4,13,18,19,27,28

The use of the deltoid splitting mini-open approach allows for surgical fixation of the rotator cuff without disruption of the deltoid origin, and enables an earlier initiation of passive and active range of motion during postsurgical rehabilitation, as compared to rehabilitation following a technique of open rotator cuff repair where the deltoid origin is detached to allow for greater surgical exposure., 8 Additionally, the use of arthroscopy prior to mini-open repair allows orthopaedic surgeons to evaluate the integrity of the glenoid labrum and other intraarticular structures that can be addressed to restore normal anatomy. ${ }^{9}$ 
In addition to long-term follow-up, more immediate short-term objective information can be used to assist physical therapists in understanding the rate of range of motion and muscular strength return, as well as the perceived level of function by the patient. This information can be used to provide realistic goal setting by the therapist and provide valuable information to patient's regarding expected return to functional activity, as well as the average time frame for the return of range of motion and strength. The purpose of this descriptive study was to objectively measure and report shoulder range of motion and strength following mini-open rotator cuff repair and rehabilitation using a standardized protocol.

\section{METHODS}

\section{Patients}

A retrospective chart review was undertaken of patients who underwent an arthroscopically assisted mini-open repair of a full thickness rotator cuff tear and were referred to Physiotherapy Associates Scottsdale Sports Clinic over a 3-year period for rehabilitation by the senior author (T.E.), using our institution's standard rehabilitation protocol (Appendix). Subjects were not included in the chart review if concomitant procedures were performed during surgery, including labral repair, thermal capsulorrhaphy, or capsular placation. To be included in this study, subjects needed to be free of pathology or surgical procedures on the contralateral extremity. Subjects who had either a partial repair of the full-thickness tear or a massive tear $(>5 \mathrm{~cm})$ were excluded from this study. All tears were classified as small $(1-2 \mathrm{~cm})$, medium $(2-3 \mathrm{~cm})$, or large $(3-5 \mathrm{~cm})$ for inclusion in this study. The research procedure was reviewed and approved by the Institutional Review Board of Physiotherapy Associates (Memphis, TN).

\section{Rehabilitation Program}

Additional information regarding the postoperative rehabilitation protocol is given here to provide insight into the specific treatment each patient received following the mini-open rotator cuff repair. Sling use was directed by the physician, with the recommendation for use in precarious situations, such as when outside the home or work environment, and to provide comfort. No specific objective criteria were used to monitor and direct sling use. Beginning in the first week postsurgery (stomach rubs and pendulum exercises) and increasing during the first postoperative physical therapy visit (overhead pulley, supine flexion, and scaption with a cane), patients began active-assisted shoulder range-of-motion exercises. McCann et $\mathrm{al}^{21}$ has shown, using EMG, significant increases in muscular activity between supine forward- flexion assisted exercise and use of the overhead pulley in a seated position in the deltoid and rotator cuff musculature. Our protocol combines the supine antigravity work and the seated against-gravity work with a pulley for range-of-motion recovery.

Early passive shoulder range of motion and glenohumeral joint mobilization was initiated in all planes of motion. ${ }^{16}$ Cadaveric research has shown that rotational range of motion performed in the scapular or coronal plane does not increase tissue tension that would prove deleterious to the rotator cuff repair. ${ }^{16}$ These early range-of-motion strategies are performed both in-clinic on a 3-times-weekly basis, as well as multiple (2-3) times daily at home by the patient.

In the early postoperative rehabilitation period is the inclusion of manual resistance for the motions of scapular protraction and retraction. Active shoulder motion is also initiated using the balance point position of the shoulder. In this position (supine with the shoulder in $90^{\circ}$ of flexion) the patient is able to control the extremity with minimal muscular activation. This position is used to perform small active movements of flexion/extension and horizontal abduction/adduction, with the therapist guiding the patients range of motion. The balance point position is also used to initiate rhythmic stabilization exercises using the command "hold don't let me move you." With this exercise the patient holds the $90^{\circ}$ shoulder flexion position against external challenges in multiple directions of movement by the therapist. ${ }^{29} \mathrm{~A}$ position of scapular protraction is employed with this exercise to increase activation of the serratus anterior. $^{7,24}$

During weeks 5 and 6 , submaximal resistive exercise is initiated. The primary movements are shoulder internal and external rotation, and shoulder extension. These movements are targeted due to activation of the infraspinatus, teres minor, and subscapularis, and protective positions below $90^{\circ}$ of shoulder elevation and short lever arms to apply resistance are used. ${ }^{26,31}$ Use of low-resistance (tan) Thera-Band tubing (Hygenic Corp, Akron, $\mathrm{OH}$ ) and/or little or no added weight to the extremity is recommended. Caution should be given regarding the use of heavyresistance exercise and long lever arms, as experimental research has shown that even with submaximal cyclic loading, rotator cuff tendon failure can occur. ${ }^{5}$ Moncrief et $\mathrm{al}^{22}$ have shown how the use of these low-resistance exercises can lead to increases in rotator cuff strength using a high-repetition format recommended in this protocol.

At 10 to 12 weeks postsurgery patients started using an isokinetic dynamometer at submaximal intensities. The accommodative resistance, ability to exercise via a sequence of progressively greater movement velocities, and objective feedback all provide the rationale for inclusion of this type of resistive exercise. ${ }^{10,11}$ 
Additionally, several studies have reported significant increases in glenohumeral joint internal and external rotation strength following isokinetic training. ${ }^{11,23}$

\section{Outcome Measures}

Variables included in the retrospective review were subject age, dominant arm, estimated time from injury to surgery, time from surgery to initiation of physical therapy, as well as objective measures of shoulder range of motion and strength. Range of motion was initially measured passively in the supine position at 6 weeks postsurgery for forward flexion and abduction, with a universal goniometer and standardized measurement techniques. ${ }^{25}$ Internal and external rotation was measured at $90^{\circ}$ of shoulder abduction while providing scapular stabilization.,12 At 12 weeks postsurgery, shoulder active range of motion was measured against gravity with the subject in a seated position for forward elevation and abduction. Supine shoulder active internal and external rotation at $90^{\circ}$ of abduction was also measured while providing scapular stabilization. ${ }^{12}$ The identical procedures were used to document active range of motion of the uninjured shoulder during the initial postoperative evaluation. Measurements were recorded to the nearest degree. All measures were taken by the senior author as part of the rehabilitation process. Test-retest reliability (Pearson correlation) results of the glenohumeral joint rotational measures published previously and reported by these authors were $r=0.94$ for external rotation and $r=$ 0.89 for internal rotation. ${ }^{12}$

Isokinetic strength testing was performed on 28 of the 37 patients 12 weeks postsurgery using a Cybex 6000 Isokinetic dynamometer. Isokinetic strength testing was not performed on all 37 patients due to early discharge from therapy and/or patient noncompliance in 9 patients. Isokinetic data were obtained in 5 of these 9 patients at time intervals less than 10 weeks postsurgery, or greater than 14 weeks postsurgery. Due to the retrospective nature of the study, exact timing of the isokinetic internal/external rotation test in these 9 patients did not allow those data to be included in the analysis. Testing was performed with the patient in a standing position with the dynamometer placed in the modified base position. This position utilizes $30^{\circ}$ of tilt of the dynamometer from the horizontal base position and places the patient's shoulder in $30^{\circ}$ of elevation in the scapular plane. ${ }^{6} \mathrm{~A}$ range of motion of $70^{\circ}$ of internal rotation and $30^{\circ}$ of external rotation was set using range-of-motion stops. Four progressively more difficult (50\%, 75\%, $90 \%$, and $100 \%$ of maximum) submaximal warm-up repetitions were used, followed by 5 maximal-effort repetitions for data collection at the testing speeds of $90^{\circ} \mathrm{s}, 210^{\circ} / \mathrm{s}$, and $300^{\circ} / \mathrm{s}$, with 30 seconds rest between testing speeds. Testing was performed at 3 test speeds to describe the patient's ability to gener- ate torque at a slow, intermediate, and a fast angular velocity. Testing was performed on the uninjured extremity first, without randomization of testing speed sequence to enhance reliability. ${ }^{33}$ Following testing on the uninjured extremity, identical setup and testing procedures were used on the postoperative extremity. The isokinetic parameter chosen to represent muscular performance in this population was the single-repetition work value calculated by the Cybex 6000 software, as the area under the torque curve versus joint angle curve for the best repetition performed by the subject. Additionally, the external/ internal rotation unilateral single-repetition work ratio was recorded as calculated by the Cybex 6000 software by dividing the external rotation work value obtained at each speed for the best repetition by the corresponding internal rotation work value. In previous studies the reliability of the Cybex isokinetic dynamometer has been reported $(\mathrm{ICC}=0.83-0.99)^{30}$ as has the reliability specific to the application of isokinetic testing to the glenohumeral joint (ICC $=$ $0.60-0.95) .^{20}$

The self-report section of the modified American Shoulder Elbow Surgeons (ASES) Rating Scale was administered at 12 weeks postsurgery (Figure)., ${ }^{2,3}$ Patients answered the series of 15 questions following standardized instructions estimating their ability to perform the activities with their injured extremity at the time the instrument was completed. Each patient's responses were tallied to form a composite score against 45 possible points. The higher a score, the greater level of function that score represents. The Modified ASES Rating Scale has been studied to assess its test-retest reliability and responsiveness in patients with shoulder pain. ${ }^{2,3}$ The ICC was 0.96 and a standardized response mean of 0.93 was reported., ${ }^{2,3}$ The Modified ASES Rating Scale compared favorably to other shoulder rating scales and was found to be more sensitive to change than a generic questionnaire. $^{3}$

\section{Data Analysis}

SPSS (Chicago, IL) was used to generate descriptive statistics on the variables measured in this investigation.

\section{RESULTS}

The mean $( \pm \mathrm{SD})$ age of the 37 patients (11 female and 26 male) studied was $57.3 \pm 9.9$ years. The mean $( \pm \mathrm{SD})$ time from initial injury to surgical repair of the rotator cuff was $11.8 \pm 9.1$ weeks, with a range of 2 to 35 weeks. Patients were seen for their first visit of physical therapy and evaluated at a mean $( \pm \mathrm{SD})$ of $3.0 \pm 2.0$ weeks postsurgery. Surgery was performed on

J Orthop Sports Phys Ther • Volume $36 \bullet$ Number $5 \bullet$ May 2006 


\section{MODIFIED AMERICAN SHOULDER \& ELBOW SURGEONS RATING SCALE}

\section{Please rate your ability to do the following daily activities using the following scale:}

$$
0=\text { unable, } 1=\text { very difficult, } 2=\text { somewhat difficult, } 3=\text { not difficult at all. }
$$

\section{Getting dressed including} putting on your coat

Wash back/do up bra

Manage toileting

\section{Comb hair}

Reach a high shelf

Lifting heavy objects

Do usual work

Do usual sport

Sleep on your painful side

Throw a ball overhand

Open jar of food

Cut with a knife

Use a phone

Do up buttons

Carry shopping bag

FIGURE. Self-report section of the modified American Shoulder and Elbows Surgeons Rating Scale.

the dominant arm in 28 of 37 cases. Patients were typically seen 2 to 3 times per week for physical therapy, with a mean $( \pm \mathrm{SD})$ number of $10.0 \pm 5.8$ visits from the initial evaluation to the 6-week postsurgery stage, and mean $( \pm \mathrm{SD})$ of $13.5 \pm 5.5$ visits between 6 and 12 weeks postsurgery.

Passive range-of-motion measurements taken 6 weeks following mini-open repair of the rotator cuff are presented in Table 1 . In addition, the active range-of-motion values of the contralateral extremity taken during the initial evaluation are listed for reference. The movement of forward flexion at 6 weeks shows the smallest mean difference relative to the uninjured extremity, with abduction and external rotation having the largest deficiencies.
Table 2 contains the active range-of-motion measures taken at 12 weeks following mini-open repair, as well as the number of degrees of difference relative to the uninjured extremity. Values obtained for forward flexion on the involved extremity exceeded values from the contralateral uninjured extremity. Mean deficits in range of motion at 12 weeks postoperatively were $5^{\circ}$ to $7^{\circ}$ for abduction and internal and external rotation measured at $90^{\circ}$ of abduction.

Table 3 contains the isokinetic single-repetition work values, as well as isokinetic bilateral strength comparisons, expressed as the percent deficit of the injured extremity relative to the uninjured extremity for shoulder internal and external rotation at the 3 
testing speeds. Results show deficits of $5 \%$ to $7 \%$ for the external rotators compared to the uninvolved extremity. Internal rotation strength in the involved extremity was $6 \%$ to $11 \%$ stronger compared to the uninvolved side at the faster contractile velocities. Table 4 contains the external/internal rotation work ratios for the injured and uninjured extremity. Mean external/internal rotation work ratios on the injured extremity ranged between $45 \%$ and $60 \%$, with corresponding mean values on the uninjured extremity ranging between $58 \%$ and $64 \%$.

The self-report section of the Modified ASES Rating Scale administered at 12 weeks postsurgery produced mean values of 38.7 out of 45.0 possible points. $^{2,3}$

\section{DISCUSSION}

This study provides descriptive information on the short-term outcome following a common surgical procedure seen in orthopaedic and sports physical therapy clinics. These measures taken while the

TABLE 1. Mean \pm SD (range) passive range of motion (PROM) of the involved extremity 6 weeks following mini-open rotator cuff repair and active range of motion (AROM) of the uninvolved extremity taken at initial examination ( $n=37$ patients).

\begin{tabular}{lcc}
\hline \multicolumn{1}{c}{ Movement } & $\begin{array}{c}\text { PROM Injured } \\
\text { Extremity }\end{array}$ & $\begin{array}{c}\text { AROM Uninjured } \\
\text { Extremity }\end{array}$ \\
\hline Forward flexion $\left(^{\circ}\right)$ & $154 \pm 16$ & $156 \pm 11$ \\
$(115$ to 175$)$ & $(138$ to 177$)$ \\
Abduction $\left(^{\circ}\right)$ & $138 \pm 24$ & $164 \pm 12$ \\
$(85$ to 175$)$ & $(125$ to 183$)$ \\
External rotation at & $74 \pm 19$ & $91 \pm 9$ \\
$90^{\circ}$ abduction $\left(^{\circ}\right)$ & $(30$ to 100$)$ & $(65$ to 105$)$ \\
Internal rotation at & $39 \pm 12$ & $48 \pm 12$ \\
$90^{\circ}$ abduction $\left(^{\circ}\right)$ & $(15$ to 60$)$ & $(24$ to 70$)$ \\
\hline
\end{tabular}

TABLE 2. Mean $\pm S D$ (range) active range of motion of the involved extremity 12 weeks following mini-open rotator cuff repair ( $n=37$ patients). A negative value indicates injured extremity exceeds value measured on uninjured extremity.

\begin{tabular}{ccc}
\hline Movement & Injured Extremity & $\begin{array}{c}\text { Difference } \\
\text { from Uninjured } \\
\text { Extremity }\end{array}$ \\
\hline Forward flexion $\left(^{\circ}\right)$ & $\begin{array}{c}165 \pm 14 \\
(125 \text { to } 180)\end{array}$ & $\begin{array}{c}-9 \pm 16 \\
(-38 \text { to } 35)\end{array}$ \\
Abduction $\left(^{\circ}\right)$ & $156 \pm 21$ & $7 \pm 23$ \\
& $(80$ to 180$)$ & $(-30$ to 90$)$ \\
External rotation at & $86 \pm 9$ & $5 \pm 18$ \\
$90^{\circ}$ abduction $\left(^{\circ}\right)$ & $(60$ to 105$)$ & $(-25$ to 40$)$ \\
Internal rotation at & $43 \pm 8$ & $5 \pm 17$ \\
$90^{\circ}$ abduction $\left(^{\circ}\right)$ & $(24$ to 60$)$ & $(-25$ to 45$)$ \\
\hline
\end{tabular}

TABLE 3. Isokinetic shoulder internal (IR) and external rotation (ER) single-repetition work values and injured/uninjured comparisons measured at 12 weeks status after mini-open rotator cuff repair ( $n=28$ subjects). All measures expressed in footpounds $(1 \mathrm{ft}-\mathrm{lb}=2.98 \mathrm{Nm})$.

\begin{tabular}{|c|c|c|c|}
\hline $\begin{array}{l}\text { Motion and } \\
\text { Speed }\end{array}$ & $\begin{array}{c}\text { Injured } \\
(\text { Mean } \pm \text { SD })\end{array}$ & $\begin{array}{c}\text { Uninjured } \\
(\text { Mean } \pm \text { SD) }\end{array}$ & $\begin{array}{l}\text { Percent Deficit } \\
(\text { Mean } \pm \text { SD })^{*}\end{array}$ \\
\hline ER $90 \% \mathrm{~s}$ & $20.9 \pm 7.9$ & $23.3 \pm 9.3$ & $\begin{array}{l}7.4 \pm 25.0 \\
(41 \text { to }-75)\end{array}$ \\
\hline ER $210 \% / s$ & $14.2 \pm 6.0$ & $15.7 \pm 7.1$ & $\begin{array}{l}5.1 \pm 26.0 \\
(45 \text { to }-80)\end{array}$ \\
\hline ER $300 \%$ s & $9.3 \pm 4.9$ & $10.7 \pm 6.1$ & $\begin{array}{l}6.9 \pm 44.0 \\
(79 \text { to }-100)\end{array}$ \\
\hline IR $90^{\circ} / \mathrm{s}$ & $35.5 \pm 14.4$ & $36.7 \pm 13.7$ & $\begin{array}{c}2.1 \pm 26.0 \\
(39 \text { to }-89)\end{array}$ \\
\hline IR $210 \% / s$ & $28.0 \pm 11.6$ & $27.5 \pm 12.4$ & $\begin{array}{r}-6.0 \pm 029.0 \\
(41 \text { to }-100)\end{array}$ \\
\hline IR $300 \%$ s & $20.8 \pm 11.3$ & $19.7 \pm 11.3$ & $\begin{array}{l}-11.1 \pm 55.0 \\
(60 \text { to }-229)\end{array}$ \\
\hline
\end{tabular}

* Negative value indicates involved extremity is stronger than uninvolved. Range in parentheses.

TABLE 4. Shoulder external/internal rotation isokinetic work ratios measured at 12 weeks following mini-open rotator cuff repair ( $n=28$ subjects).

\begin{tabular}{llc}
\hline Speed & $\begin{array}{c}\text { Injured Shoulder } \\
\text { (Mean } \pm \text { SD } \\
\text { [Range]) }\end{array}$ & $\begin{array}{c}\text { Uninjured Shoulder } \\
\text { (Mean } \pm \text { SD } \\
\text { [Range]) }\end{array}$ \\
\hline $90 \% \mathrm{~s}$ & $60 \pm 9(45-80)$ & $63 \pm 11(37-85)$ \\
$210 \% \mathrm{~s}$ & $51 \pm 11(31-80)$ & $58 \pm 17(29-100)$ \\
$300 \% \mathrm{~s}$ & $46 \pm 14(27-100)$ & $57 \pm 19(20-114)$ \\
\hline
\end{tabular}

patient was under the direct care of the therapist can be used to guide the clinical decision-making process. For example, patients who present at 6 weeks postsurgery with limited passive range of motion should be targeted for additional emphasis on rangeof-motion exercises and more aggressive mobilization and clinical stretching techniques. ${ }^{9}$ In contrast, patients who present with the expected amount of passive range of motion can spend less valuable home exercise time on range-of-motion activities and focus more on strengthening the rotator cuff and scapular musculature. ${ }^{9}$

The range-of-motion findings reported in this patient series suggest the value of limited immobilization and early range-of-motion exercises and limited immobilization following mini-open rotator cuff repair. Patients were started in physical therapy a mean of 3 weeks postoperatively. The application of a protocol of rehabilitation that includes early passive and active-assisted range-of-motion exercises in the first 6 weeks resulted in passive shoulder flexion range-of-motion values for the postsurgical side nearly equal to those of the uninjured extremity. Cadaveric research by Hatakeyama et $\mathrm{al}^{16}$ provides guidance to clinicians working with patients following rotator cuff repair by outlining the inherent tension in the 
repaired tendon with passive movements typically performed in postoperative physical therapy. These authors state that external rotation range of motion of up to $60^{\circ}$ actually decreases the tension in the repaired supraspinatus tendon when performed in the coronal or scapular plane.

Additionally, the active range-of-motion measures taken at 12 weeks postsurgery indicated a full return of forward flexion movement and deficits of only $7^{\circ}$ in abduction and less than $5^{\circ}$ for internal and external rotation, as compared to the contralateral extremity.

Gore et $\mathrm{al}^{14}$ measured active range of motion in 58 patients, with a mean age of 62 years, who underwent an open rotator cuff repair. Patients were tested a mean of 5.5 years following surgery and had undergone postoperative rehabilitation following 4 to 6 weeks of sling immobilization. Pendulum exercises were performed at 4 weeks postoperatively, with active range of motion starting after 6 weeks postoperatively. No data were reported for the uninvolved extremity; however, active range of motion 5.5 years following surgery was $126^{\circ}$ of flexion, $130^{\circ}$ of abduction, $66^{\circ}$ of external rotation measured at $90^{\circ}$ of abduction, and $42^{\circ}$ of internal rotation measured at $90^{\circ}$ of abduction. The patients studied by Gore et $\mathrm{al}^{14}$ had longer periods of immobilization; however, those patients underwent a surgical procedure where detachment of the deltoid was carried out, which may have further compromised the range-of-motion return as compared to the subjects in the present study, who underwent a mini-open rotator cuff repair.

The isokinetic muscular strength comparisons at 12 weeks postsurgery indicate deficits in shoulder external rotation strength at all 3 testing speeds. These deficits in single-repetition work values ranged between $5 \%$ to $7 \%$. Comparison to other studies using objective testing in patients following rotator cuff repair is difficult, as many long-term studies rely on rating scales and manual muscle testing to measure outcome. Isokinetic evaluation of muscular strength at 6 months and 1 year following open rotator cuff repair using an open deltopectoral approach, with deltoid detachment in 15 subjects and a mini-open deltoid splitting approach in 10 subjects, using a Cybex isokinetic dynamometer, was performed by Kirschenbaum et al. ${ }^{19}$ At 6 months postoperatively, testing was performed bilaterally at only 1 speed $\left(90^{\circ} / \mathrm{s}\right)$, with deficits in external rotation measured at $24 \%$. By 1 year postoperatively, the involved extremity had achieved a level of strength $42 \%$ greater than the uninjured extremity for external rotation. Deficits in external rotation strength measured at only 3 months postoperatively in this study are moderately less than those reported by Kirschenbaum et al ${ }^{19}$ at 6 months postsurgery. The fact that greater than half of the patients in the Kirschenbaum et $\mathrm{al}^{19}$ study had a surgical exposure with deltoid detachment may have been one factor that slowed the return of strength. Despite the presence of deficits of the external rotators at 6 months postsurgery in the study by Kirschenbaum et al, ${ }^{19}$ their patients achieved a level of strength actually $42 \%$ greater than the uninjured extremity by 1 year following surgery.

Rokito et $\mathrm{al}^{27}$ studied 42 consecutive patients following a traditional open rotator cuff repair that included elevation of the anterior deltoid for surgical exposure. Strength was measured using a Biodex isokinetic dynamometer for flexion, abduction, and external rotation at $60^{\circ} \mathrm{s}$ at $3,6,9$, and 12 months postsurgery. External rotation peak torque comparisons between the injured and uninjured extremity were $59 \%, 68 \%, 73 \%$, and $90 \%$, respectively, during the postoperative course. Similar to the findings reported by Kirschenbaum et al, ${ }^{19}$ Rokito et $\mathrm{al}^{27}$ showed greater external rotation strength deficiencies at 3 months postsurgery following an open rotator cuff repair compared to the present results of this study examining the mini-open rotator cuff surgical approach. Further research and follow-up is needed on our patients following the mini-open rotator cuff repair to allow a comparison to the long-term strength data provided by both Kirschenbaum et $\mathrm{al}^{19}$ and Rokito et al. ${ }^{27}$

While deficits in external rotation of $5 \%$ to $7 \%$ were identified with bilateral comparison at 3 months postsurgery, a clinically significant isokinetic finding identified in this study was the lower external/ internal rotation ratio on the operative extremity. The external/internal rotation ratio is used to estimate the degree of muscular balance between the larger anterior muscle groups (internal rotators) and the posterior rotator cuff. ${ }^{6,10}$ External/Internal rotation ratios measured in normal healthy subjects has been reported at $66 \%$ at multiple contractile velocities. ${ }^{6,8,10,17}$ Ratios below this $66 \%$ standard indicate a relative imbalance of the posterior rotator cuff. ${ }^{32}$ The ratios measured in this study show the postoperative extremity to be approximately $3 \%$ to $11 \%$ lower on the injured extremity. This would indicate the need for continued strengthening of the external rotators to normalize this ratio. ${ }^{34,35}$

Additional research demonstrating the importance of the external/internal rotation unilateral strength ratio in patients with rotator cuff disease was presented by Wilk et al. ${ }^{34}$ Patients following rotator cuff tears were unable to elevate their upper extremity actively against gravity until a mean external/internal rotation unilateral strength ratio greater than 52\% was achieved. The continued application of resistive exercise to achieve greater muscle balance is a characteristic of the postoperative protocol used in this study as well as in other postoperative protocols following rotator cuff repair. ${ }^{1}$

This study has several limitations. We only examined the objective parameters of range of motion, 
muscular strength, and patient perceived satisfaction via a rating instrument, and therefore cannot comment or provide insight into other factors in the recovery following mini-open rotator cuff repair. Additionally, the study was retrospective. These authors recommend additional research, prospective in design, with longer follow-ups, to better understand the complete recovery following this surgical procedure.

\section{CONCLUSION}

The data collected following mini-open rotator cuff repair indicate a $5^{\circ}$ to $7^{\circ}$ mean deficit in shoulder abduction and internal/external rotation active range of motion, with a full return of forward flexion 12 weeks following surgery. Isokinetic strength testing shows a $5 \%$ to $7 \%$ deficit in external rotation strength, with $6 \%$ to $11 \%$ greater internal rotation strength on the postsurgical side at intermediate and fast contractile velocities. Self-reported data from the Modified ASES Rating Scale showed patients to score $38.7 / 45.0$ points.

\section{REFERENCES}

1. Andrews JA, Wilk KE. The Athlete's Shoulder. New York, NY: Churchill Livingstone; 1994.

2. Beaton D, Richards RR. Assessing the reliability and responsiveness of 5 shoulder questionnaires. J Shoulder Elbow Surg. 1998;7:565-572.

3. Beaton DE, Richards RR. Measuring function of the shoulder. A cross-sectional comparison of five questionnaires. J Bone Joint Surg Am. 1996;78:882-890.

4. Blevins FT, Warren RF, Cavo C, et al. Arthroscopic assisted rotator cuff repair: results using a mini-open deltoid splitting approach. Arthroscopy. 1996;12:50-59.

5. Burkhart SS, Diaz Pagan JL, Wirth MA, Athanasiou KA. Cyclic loading of anchor-based rotator cuff repairs: confirmation of the tension overload phenomenon and comparison of suture anchor fixation with transosseous fixation. Arthroscopy. 1997;13:720-724.

6. Davies GJ. A Compendium of Isokinetics in Clinical Usage. LaCrosse, WI: S \& S Publishers; 1992.

7. Decker MJ, Hintermeister RA, Faber KJ, Hawkins RJ. Serratus anterior muscle activity during selected rehabilitation exercises. Am J Sports Med. 1999;27:784-791.

8. Ellenbecker TS. Clinical Examination of the Shoulder. Philadelphia, PA: Elsevier Science; 2004.

9. Ellenbecker TS, Bailie DS, Kibler WB. Rehabilitation Following Mini-Open and Arthroscopic Rotator Cuff Repairs. In: Manske R, ed. Post-Operative Rehabilitation of the Knee and Shoulder. Philadelphia, PA: Elsevier Science; 2005:

10. Ellenbecker TS, Davies GJ. The application of isokinetics in testing and rehabilitation of the shoulder complex. J Athl Train. 2000;35:338-350.

11. Ellenbecker TS, Davies GJ, Rowinski MJ. Concentric versus eccentric isokinetic strengthening of the rotator cuff. Objective data versus functional test. Am J Sports Med. 1988;16:64-69.

12. Ellenbecker TS, Roetert EP, Piorkowski PA, Schulz DA. Glenohumeral joint internal and external rotation range of motion in elite junior tennis players. J Orthop Sports Phys Ther. 1996;24:336-341.

13. Gazielly DF, Gleyze P, Montagnon C. Functional and anatomical results after rotator cuff repair. Clin Orthop Relat Res. 1994;43-53.

14. Gore DR, Murray MP, Sepic SB, Gardner GM. Shouldermuscle strength and range of motion following surgical repair of full-thickness rotator-cuff tears. J Bone Joint Surg Am. 1986;68:266-272.

15. Hata Y, Saitoh S, Murakami N, Kobayashi H, Takaoka K. Atrophy of the deltoid muscle following rotator cuff surgery. J Bone Joint Surg Am. 2004;86-A:1414-1419.

16. Hatakeyama Y, Itoi E, Urayama M, Pradhan RL, Sato K. Effect of superior capsule and coracohumeral ligament release on strain in the repaired rotator cuff tendon. A cadaveric study. Am J Sports Med. 2001;29:633-640.

17. Ivey FM, Jr., Calhoun JH, Rusche K, Bierschenk J. Isokinetic testing of shoulder strength: normal values. Arch Phys Med Rehabil. 1985;66:384-386.

18. Kim SH, Ha KI, Park JH, Kang JS, Oh SK, Oh I. Arthroscopic versus mini-open salvage repair of the rotator cuff tear: outcome analysis at 2 to 6 years' follow-up. Arthroscopy. 2003;19:746-754.

19. Kirschenbaum D, Coyle MP, Jr., Leddy JP, Katsaros P Tan F, Jr., Cody RP. Shoulder strength with rotator cuff tears. Pre- and postoperative analysis. Clin Orthop Relat Res. 1993;174-178.

20. Malerba JL, Adam ML, Harris BA, Krebs DE. Reliability of dynamic and isometric testing of shoulder external and internal rotators. J Orthop Sports Phys Ther. 1993; 18:543-552.

21. McCann PD, Wootten ME, Kadaba MP, Bigliani LU. A kinematic and electromyographic study of shoulder rehabilitation exercises. Clin Orthop Relat Res. 1993;179-188.

22. Moncrief SA, Lau JD, Gale JR, Scott SA. Effect of rotator cuff exercise on humeral rotation torque in healthy individuals. J Strength Cond Res. 2002;16:262-270.

23. Mont MA, Cohen DB, Campbell KR, Gravare K, Mathur SK. Isokinetic concentric versus eccentric training of shoulder rotators with functional evaluation of performance enhancement in elite tennis players. Am J Sports Med. 1994;22:513-517.

24. Moseley JB, Jr., Jobe FW, Pink M, Perry J, Tibone J. EMG analysis of the scapular muscles during a shoulder rehabilitation program. Am J Sports Med. 1992;20:128134.

25. Norkin CC, White DJ. Measurement of Joint Motion: A Guide to Goniometry. 2nd ed. Philadelphia, PA: F.A. Davis Company; 1995.

26. Reinold MM, Wilk KE, Fleisig GS, et al. Electromyographic analysis of the rotator cuff and deltoid musculature during common shoulder external rotation exercises. J Orthop Sports Phys Ther. 2004;34:385-394.

27. Rokito AS, Zuckerman JD, Gallagher MA, Cuomo F. Strength after surgical repair of the rotator cuff. J Shoulder Elbow Surg. 1996;5:12-17.

28. Severud EL, Ruotolo C, Abbott DD, Nottage WM All-arthroscopic versus mini-open rotator cuff repair: A long-term retrospective outcome comparison. Arthroscopy. 2003;19:234-238.

29. Sullivan PE, Markos PD, Minor MD. An Integrated Approach to Therapeutic Exercise: Theory and Clinical Application. Reston, VA: Reston Publishing Co; 1982.

30. Timm KE, Genrich P, Bruns R, Fyke D. The mechanical and physiological performance reliability of selected isokinetic dynamometers. Isokinet Exerc Sci. 1992;2:182-190. 
31. Townsend $H$, Jobe FW, Pink M, Perry J. Electromyographic analysis of the glenohumeral muscles during a baseball rehabilitation program. Am J Sports Med. 1991;19:264-272.

32. Warner JJ, Micheli LJ, Arslanian LE, Kennedy J, Kennedy R. Patterns of flexibility, laxity, and strength in norma shoulders and shoulders with instability and impingement. Am J Sports Med. 1990;18:366-375.
33. Wilhite MR, Cohen ER, Wilhite SC. Reliability of concentric and eccentric measurements of quadriceps performance using the Kin-Com dynamometer. The effects of testing order for three different speeds. J Orthop Sports Phys Ther. 1992;15:175-182.

34. Wilk KE, Arrigo C. Current concepts in the rehabilitation of the athletic shoulder. J Orthop Sports Phys Ther. 1993;18:365-378. 


\section{Appendix}

\section{Postoperative Rehabilitation Protocol for Mini-Open Rotator Cuff Repair Using Deltoid Splitting Approach}

\section{General Guidelines}

- Progression of resistive exercise and range of motion (ROM) is dependent on patient tolerance

- Resistance exercise should not be performed with specific shoulder joint pain or pain over the incision site

- A sling is provided to the patient for support as needed with daily activities and to wear at night. The patient is weaned from the sling as tolerated

- Early home exercises given to the patient following surgery include stomach rubs, sawing, and gripping activity

- Progression to active range of motion (AROM) against gravity and duration of sling use is predicated both on the size of the rotator cuff tear and quality of the tissue, and guided by the referring physician

\section{Postoperative Weeks 1 and 2}

1. Early passive range of motion (PROM) to patient tolerance during the first 4 to 6 weeks

a. Flexion

b. Scapular and coronal plane abduction

c. Internal rotation/external rotation (IR/ER) performed between $90^{\circ}$ to $45^{\circ}$ shoulder abduction

2. Submaximal isometric IR/ER, flexion/extension, and adduction

3. Mobilization of the glenohumeral joint and scapulothoracic joint. Passive stretching of elbow, forearm, and wrist to terminal ranges

4. Side-lying scapular protraction/retraction resistance to encourage early serratus anterior and lower trapezius activation and endurance

5. Home exercise instruction

a. Instruction in PROM and active-assisted range-of-motion (AAROM) home exercises with T-bar, pulleys, or opposite-arm assistance in supine position using ROM to patient tolerance

b. Weight-bearing Codman exercise instruction over a ball or countertop/table

c. Theraputty for grip strength maintenance

\section{Postoperative Week 3}

1. Continue above shoulder ROM and isometric strength program to patient tolerance. Progress patient to AAROM exercises

2. Add upper-body ergometer (UBE) if available

3. Begin active scapular strengthening exercises and continue side-lying manual scapular stabilization exercise

a. Scapular retraction

b. Scapular retraction with depression

4. Begin resistive exercise for total arm strength using positions with glenohumeral joint completely supported including

a. Bicep curls

b. Tricep curls

c. Wrist curls: flexors, extensors, radial, and ulnar deviators

5. Begin submaximal rhythmic stabilization using the balance point position (patient in supine position with $90^{\circ}$ to $100^{\circ}$ of shoulder elevation) to initiate dynamic stabilization

\section{Postoperative Weeks 5 and 6}

1. Initiate isotonic resistance exercise focusing on the following movements

a. Side-lying ER

b. Prone extension

c. Prone horizontal abduction (limited range to $45^{\circ}$ )

d. Supine IR

e. Flexion to $90^{\circ}$

(Note: A low-resistance/high-repetition [ie, 30 reps] format is recommended, using no resistance initially [ie, weight of the arm])

2. Progression to full PROM and AROM in all planes including ER and IR in neutral adduction, progressing from the $90^{\circ}$ abducted position used initially postsurgery

3. ER oscillation (resisted ER with towel roll under axilla and oscillation device)

4. Home exercise program for strengthening the rotator cuff and scapular musculature with weights and/or elastic tubing (Thera-Band)

\section{Postoperative Week 8}

1. Begin weight bearing step-ups and quadruped rhythmic stabilization exercise

2. Initiate upper extremity plyometric chest passes and functional 2-hand rotation tennis groundstroke or golf swing simulation using small exercise ball, progressing to light medicine ball as tolerated 


\section{Postoperative Week 10}

1. Initiation of submaximal isokinetic exercise for IR/ER in the modified neutral position (Note: Criterion for progression to isokinetic exercise)

a. Patient has IR and ER ROM greater than that used during the isokinetic exercise

b. Patient can complete isotonic exercise program pain free with a 1- to 1.5-kg weight or medium-resistance surgical tubing or Thera-Band

2. Progression to $90^{\circ}$ abducted rotational training in patients returning to overhead work or sport activity
a. Prone external rotation
b. Standing ER/IR rotation with $90^{\circ}$ abduction in the scapular plane
c. Statue of liberty (external rotation oscillation)

\section{Postoperative Week 12}

1. Progression to maximal isokinetics in IR/ER and isokinetic testing to assess strength in modified base 30/30/30 position. Formal documentation of AROM, PROM, and administration of shoulder rating scale

2. Begin interval return programs if criterion has been met below
IR/ER strength minimum of $85 \%$ of contralateral extremity
b. ER/IR ratio $60 \%$ or higher
c. Pain-free ROM
d. Negative impingement and instability signs during clinical exam

\section{Postoperative Week 16 (4 mo)}

1. Isokinetic re-evaluation, documentation of AROM, PROM, and Shoulder Rating Scale

2. Progression continues for return to full upper extremity sport activity (ie, throwing, serving in tennis, etc)

3. Preparation for discharge from formal physical therapy to home program phase 